\title{
Catalogação de mecanismos de fecho para a produção de joias por manufatura aditiva
}

\section{Clasps mechanisms catalog for jewelry design produced by additive manufacturing}

\section{RESUMO}

Danka Farias de Oliveira dankafoliveira@gmail.com Universidade Tecnológica Federal do Paraná, Curitiba, Paraná, Brasil

\section{Fernanda Botter}

professora.botter@gmail.com

Universidade Tecnológica Federal do Paraná, Curitiba, Paraná, Brasi

Recebido: 19 ago. 2020.

Aprovado: 01 out. 2020.

Direito autoral: Este trabalho está licenciado sob os termos da Licença Creative Commons-Atribuição 4.0 Internacional.

\begin{abstract}
O presente artigo tem por objetivo descrever o processo de catalogação de mecanismos de fecho da joalheria tradicional, para que possam ser adaptados à manufatura aditiva. Esta catalogação ocorreu no âmbito do projeto de iniciação científica "Processos criativos para modelagem e fabricação de artefatos de moda utilizando a impressora 3D", que teve por resultado o projeto da plataforma MuVuCa, um banco colaborativo, aberto e gratuito, que abrigará produções de joias disponibilizadas para download, modificação e impressão. 0 método utilizado para este trabalho se caracteriza como "pesquisa para o design", tratando da coleta e processamento de dados de forma a fornecer conhecimento para o projeto, com as fases de levantamento, classificação, comparação e síntese. Como resultado, foi criado um compêndio de mecanismos, disponibilizado para consulta gratuita.
\end{abstract}

PALAVRAS-CHAVE: Moda. Projeto de Produto. Desenho Industrial.

\section{ABSTRACT}

This paper aims to describe the cataloging process of closing mechanisms of traditional jewelry, so that they can be adapted to additive manufacturing production. Such cataloging took place within the scope of the research project "Creative processes for modeling and manufacturing fashion artifacts using a 3D printer", that resulted in the design of the collaborative, open platform MuVuCa to host jewelry productions available for download, modification and printing. The method employed for this work is characterized as "Research for Design", dealing with the collection and processing of data in order to provide knowledge to subside the design process, composed by the phases of survey, classification, comparison and synthesis. As a result, we have a compendium of mechanisms, available for free consultation.

KEYWORDS: Fashion. Product Design. Industrial Design.
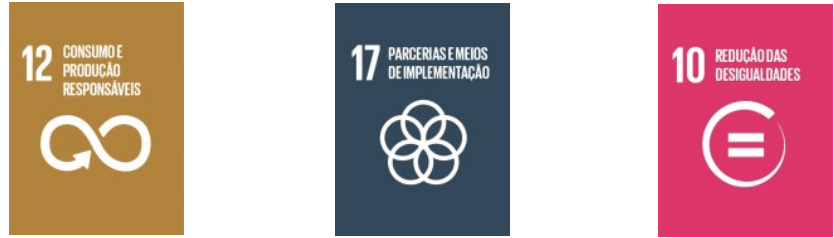


\section{INTRODUÇÃO}

Este artigo se refere a uma porção do projeto de pesquisa de Iniciação Científica intitulado "Processos criativos para modelagem e fabricação de artefatos de moda utilizando a impressora 3D", orientado pela Prof ${ }^{a} \operatorname{Dr}^{\mathrm{a}}$ Fernanda Botter, do Departamento Acadêmico de Desenho Industrial (DADIN) na Universidade Tecnológica Federal do Paraná (UTFPR). A pesquisa resultou no projeto de uma plataforma do tipo código aberto, que propõe a interação de usuários co-criadores com as modelagens de joias disponibilizadas para download, utilização e modificação. Este projeto visa a produção de design a partir do entendimento de que a prática da disciplina pode (ou deve) incluir reflexões éticas. A plataforma proposta, colaborativa e sem fins lucrativos, busca responder à pergunta de pesquisa "como o design de joias pode ser conscientizador?" De modo a entender que por uma questão de coerência, é preciso contestar a lógica capitalista de troca.

Diante desse projeto, a fase que precedeu a implementação do conceito foi dedicada à produção de alternativas. Houve a demanda de peças do tipo fechos, de forma a utilizar inspiração da joalheria tradicional para a sua adaptação para a impressão 3D.

Com dificuldade de achar literatura formal, acessível e gratuita a respeito de tais mecanismos, a pesquisa se tornou um mapeamento realizado por buscas de imagens em páginas da web.

O foco de interesse são peças que se conectam a cordas e correntes para fecho, pois foi determinado que as joias seriam compostas por pingentes combináveis. Assim, foi possível produzir um pequeno catálogo que as sintetiza, com a finalidade de orientar a co-criação das joias pelas pessoas participantes do projeto. $O$ catálogo aqui descrito encontra também seu valor como compêndio a ser consultado por pessoas interessadas no design de joias em geral.

\section{MATERIAL E MÉTODOS}

Considerando a revisão crítica da literatura em pesquisa e design, desenvolvida por Bruno Lorenz (2018), foi possível identificar a produção como "pesquisa para o design" de Findelli (1998) e Jonas (2007), por ser um subsídio ao processo projetual, ou seja, tratar da coleta e processamento de dados de forma a fornecer conhecimento para as atividades operadas. Para tanto, foram realizados os passos: levantamento (buscas em plataformas como Google Scholar ${ }^{\circledR 1}$ de pesquisa de textos acadêmicos, Pinterest ${ }^{\oplus 2}$ e Google Imagens ${ }^{\circledR 3}$ serviço de busca), classificação (feito um quadro a partir das distinções e linguagens), comparação (identificação de semelhanças e diferenças) e síntese (representação das peças que representassem as categorias mais popularizadas e distintas, feita através da ilustração digital, no programa Procreate ${ }^{\circledR 4}$ ).

\footnotetext{
${ }^{1}$ https://scholar.google.com/

2 https://br.pinterest.com/

${ }^{3}$ https://www.google.com/imghp?hl=pt-pt

4 https://procreate.art/
} 


\section{RESULTADOS}

A pesquisa se iniciou no Pinterest, para compreensão de peças de joias de forma mais visual e generalizada, com as palavras-chave "tipos de pulseira" e "tipos de colar". Através da observação de imagens, foi possível compreender que alguns colares e pulseiras não possuem fechos (são inteiriços, com tamanho ou maleabilidade o suficiente para vestir/despir), alguns são fechados com nós (usam cordas e cordões maleáveis) e alguns mais tradicionais (geralmente de bijuteria ou metais) possuem sistemas de fecho. Com isso, por serem mais abrangentes, o foco da busca foi de fechos da joalheria tradicional, que se conectam a cordas e correntes.

No Google Scholar com as palavras-chave "design de joias", "acabamento de joias" e "fechos de joias", foram feitas buscas tanto em português quanto em inglês. Foi possível encontrar apenas artigos sobre processo projetual de joias geral, mas sem a especificidade desejada. Diante desta limitação, foi recorrido o uso de plataformas como Pinterest e o Google Imagens, onde foram encontradas várias imagens ilustrativas, de direitos reservados, mas que sinalizavam os nomes de cada uma.

Com isso, foi realizada a busca com nomes encontrados específicos, de forma a identificar os equivalentes em cada língua e suas variações. As fontes encontradas foram de quatro tipos: Sites de venda como Panda Hall, Fabrique Biju, Fazendo arte Bijuterias e Natural Jóias. Sites de venda com descrições como Jewelry Supply e Fire Mountain Gems. Blogs amadores como handmade-jewelryclub e Pedra Mística. Por fim, Sites de cursos de joalheria pagos, com algumas poucas informações disponibilizadas gratuitamente como o Craftsy.

No quadro 1, foi compilado um glossário de nomenclaturas de peças encontradas com maior frequência, sinalizando suas fontes (cada uma mostrava uma seleção diferente) em língua portuguesa e inglesa, suas principais descrições de qualidades e o funcionamento das mesmas.

Aqui é possível perceber que fechos tipo springring são chamados de duas maneiras em português, assim como fechos tipo rosca são chamados de duas maneiras no inglês. Apesar de vocabulários diferenciados desenvolvidos em ambas línguas (não necessariamente frutos da tradução), existem mais nomenclaturas em inglês, algumas peças em português são apenas chamadas de "encaixe".

Quadro 1 - Dados obtidos

\begin{tabular}{|c|c|c|c|c|}
\hline $\begin{array}{l}\text { Termo usado no } \\
\text { Português }\end{array}$ & $\begin{array}{l}\text { Termo usado } \\
\text { no Inglês }\end{array}$ & Encontrado em & Funcionamento & Qualidades descritas \\
\hline $\begin{array}{l}\text { Fecho Mosquetão } \\
\text { Fecho Lagosta } \\
\text { Fecho Italiano }\end{array}$ & $\begin{array}{l}\text { Lobster claw } \\
\text { clasp } \\
\text { Swivel Clasp }\end{array}$ & $\begin{array}{c}\text { Craftsy, Pedra Mística, Handmade- } \\
\text { jewelry-club, Fire Mountain Gems, } \\
\text { Jewelry Supply, Fazendo Arte } \\
\text { Bijuterias, Natural Jóias, Fabrique } \\
\text { Biju e Panda Hall }\end{array}$ & $\begin{array}{l}\text { Apertar a alavanca } \\
\text { para que parte da } \\
\text { "garra" se abra e } \\
\text { conecte a uma argola } \\
\text { na outra extremidade } \\
\text { da corda/corrente }\end{array}$ & $\begin{array}{l}\text { Ideal para qualquer } \\
\text { colar ou pulseira até } \\
\text { porte médio }\end{array}$ \\
\hline Fecho de rosca & $\begin{array}{l}\text { Screw clasp } \\
\text { Barrel clasp } \\
\text { Torpedo clasp }\end{array}$ & $\begin{array}{c}\text { Craftsy, Pedra Mística, Fire } \\
\text { Mountain Gems, Jewelry Supply, } \\
\text { Fazendo Arte Bijuterias e Panda } \\
\text { Hall }\end{array}$ & $\begin{array}{l}\text { Manipular com as duas } \\
\text { mãos para as duas } \\
\text { peças se separarem, } \\
\text { em torção }\end{array}$ & $\begin{array}{c}\text { Aconselhável para } \\
\text { colares }\end{array}$ \\
\hline
\end{tabular}




\begin{tabular}{|c|c|c|c|c|}
\hline $\begin{array}{l}\text { Fecho T } \\
\text { Fecho de encaixe } \\
\text { redondo }\end{array}$ & $\begin{array}{l}\text { Bar and ring } \\
\quad \text { clasp } \\
\text { Toggle clasp }\end{array}$ & $\begin{array}{c}\text { Craftsy, Pedra Mística, Handmade- } \\
\text { jewelry-club, Fire Mountain Gems, } \\
\text { Jewelry Supply, Fazendo Arte } \\
\text { Bijuterias, Panda Hall }\end{array}$ & $\begin{array}{l}\text { A trava ocorre por } \\
\text { tensão entre a barra e } \\
\text { a argola, mudando a } \\
\text { posição da barra ela } \\
\text { facilmente passa por } \\
\text { dentro da argola }\end{array}$ & $\begin{array}{c}\text { Funciona bem com } \\
\text { peças pesadas, pois o } \\
\text { peso colabora com o } \\
\text { fecho }\end{array}$ \\
\hline Fecho de encaixe & $\begin{array}{l}\text { Snap clasp } \\
\text { Button clasp } \\
\text { Tab lock clasp }\end{array}$ & Fire Mountain Gems e Panda Hall & $\begin{array}{l}\text { Funcionam como um } \\
\text { botão, a peça entra por } \\
\text { meio de um furo }\end{array}$ & $\begin{array}{l}\text { Podem ser usadas } \\
\text { para peças } \\
\text { intercambiáveis }\end{array}$ \\
\hline Fecho canoa & $\begin{array}{c}\text { Ball chain } \\
\text { clasp } \\
\text { Ball-and-joint } \\
\text { clasp }\end{array}$ & $\begin{array}{c}\text { Fire Mountain Gems, Fazendo } \\
\text { Arte Bijuterias, Natural Jóias e } \\
\text { Fabrique Biju }\end{array}$ & $\begin{array}{l}\text { Usa a pressão sobre } \\
\text { uma bola para manter } \\
\text { fechado }\end{array}$ & $\begin{array}{l}\text { De fácil manuseio, } \\
\text { recomendado para } \\
\text { joias de até médio } \\
\text { porte }\end{array}$ \\
\hline $\begin{array}{l}\text { Fecho bóia } \\
\text { Fecho redondo }\end{array}$ & Springring & $\begin{array}{c}\text { Craftsy, Pedra Mística } \\
\text { Fire Mountain Gems, Jewelry } \\
\text { Supply, Fazendo Arte Bijuterias e } \\
\text { Panda Hall }\end{array}$ & $\begin{array}{l}\text { Mantém tensão por ter } \\
\text { uma mola interna, é } \\
\text { redondo e menor }\end{array}$ & $\begin{array}{l}\text { Discreto para } \\
\text { qualquer peça }\end{array}$ \\
\hline Fecho encaixe & $\begin{array}{l}\text { Fold over } \\
\text { clasp } \\
\text { Watch band } \\
\text { clasp }\end{array}$ & $\begin{array}{l}\text { Fire Mountain Gems, Natural Jóias } \\
\text { e Panda Hall }\end{array}$ & $\begin{array}{l}\text { São peças articuladas } \\
\text { para dobrar sobre si e } \\
\text { travar na extremidade }\end{array}$ & $\begin{array}{l}\text { Seguro e não enrosca } \\
\text { em outras coisas. } \\
\text { Ideal para pulseiras }\end{array}$ \\
\hline $\begin{array}{l}\text { Fecho de gancho } \\
\text { Fecho de anzol } \\
\text { Fecho elo de } \\
\text { engate }\end{array}$ & $\begin{array}{l}\text { Hook Clasp } \\
\text { Shook clasp } \\
\text { Hook and eye } \\
\text { clasp }\end{array}$ & $\begin{array}{c}\text { Craftsy, Pedra Mística, Fire } \\
\text { Mountain Gems, Jewelry Supply, } \\
\text { Fazendo Arte Bijuterias, Natural } \\
\text { Jóias e Panda Hall }\end{array}$ & $\begin{array}{c}\text { Formato de } \mathrm{S} \text {, encaixa } \\
\text { em duas argolas. Ou } \\
\text { um gancho, encaixa } \\
\text { em uma argola ou } \\
\text { outro gancho }\end{array}$ & $\begin{array}{l}\text { Indicado para } \\
\text { colares, pela } \\
\text { gravidade colaborar } \\
\text { com a trava }\end{array}$ \\
\hline $\begin{array}{l}\text { Fecho gaveta } \\
\text { Fecho caixa }\end{array}$ & $\begin{array}{l}\text { Box clasp } \\
\text { Tab insert } \\
\quad \text { clasp }\end{array}$ & $\begin{array}{l}\text { Pedra Mística, Handmade-jewelry- } \\
\text { club, Fire Mountain Gems, Natural } \\
\text { Jóias e Panda Hall }\end{array}$ & $\begin{array}{l}\text { Duas partes que se } \\
\text { encaixam como uma } \\
\text { gaveta (parte menor } \\
\text { entra na maior) }\end{array}$ & $\begin{array}{l}\text { Algumas usadas } \\
\text { para colares e } \\
\text { pulseira com mais de } \\
\text { um fio }\end{array}$ \\
\hline Fecho Gravata & $\begin{array}{l}\text { Cord locks } \\
\text { Slide clasp }\end{array}$ & $\begin{array}{l}\text { Pedra Mística, Fire Mountain } \\
\text { Gems e Panda Hall }\end{array}$ & $\begin{array}{l}\text { Uma miçanga ou conta } \\
\text { que armazena ambas } \\
\text { extremidades de uma } \\
\text { corda/fio/corrente. } \\
\text { permite ajuste } \\
\text { deslizando-a no } \\
\text { comprimento da peça }\end{array}$ & $\begin{array}{l}\text { Indicado para } \\
\text { pulseiras e colares } \\
\text { um pouco mais } \\
\text { longos, permitindo } \\
\text { customização do } \\
\text { caimento }\end{array}$ \\
\hline Fecho Magnético & $\begin{array}{l}\text { Magnetic } \\
\text { clasp }\end{array}$ & $\begin{array}{c}\text { Craftsy, Pedra Mística, Handmade- } \\
\text { jewelry-club, Fire Mountain Gems, } \\
\text { Jewelry Supply, Natural Jóias e } \\
\text { Panda Hall }\end{array}$ & $\begin{array}{l}\text { Duas peças com lados } \\
\text { opostos de ímã, que } \\
\text { naturalmente se } \\
\text { atraem }\end{array}$ & $\begin{array}{l}\text { De manuseio fácil e } \\
\text { rápido }\end{array}$ \\
\hline
\end{tabular}

Fonte: Autoria própria (2019)

Dessa maneira houve um redirecionamento para o serviço de busca Google Patentes ${ }^{\circledR 5}$, onde foram encontrados sistemas de fechos registrados tecnicamente. As criações patenteadas apresentam características únicas, mas também foi possível identificar que pareciam releituras das mais vistas na pesquisa anterior, que se diz joalheria tradicional. Por exemplo, o fecho de caixa da Patente de Jose C. Rivera (1989) parece uma representação perfeitamente semelhante ao que está em circulação no mercado atual. Como ele mesmo discorre, seu trabalho foi feito de maneira a melhorar essa espécie de peça, que frequentemente se abre.

${ }^{5}$ https://www.google.com/?tbm=pts 
Com a compreensão das peças e suas diferentes variações, foi construído este compêndio das peças mais distintas (figura 1). Inclui sinalizações de orientação de manuseio, abertura, trava e conexão a cordas e correntes.

Figura 1 - Catálogo de Fechos

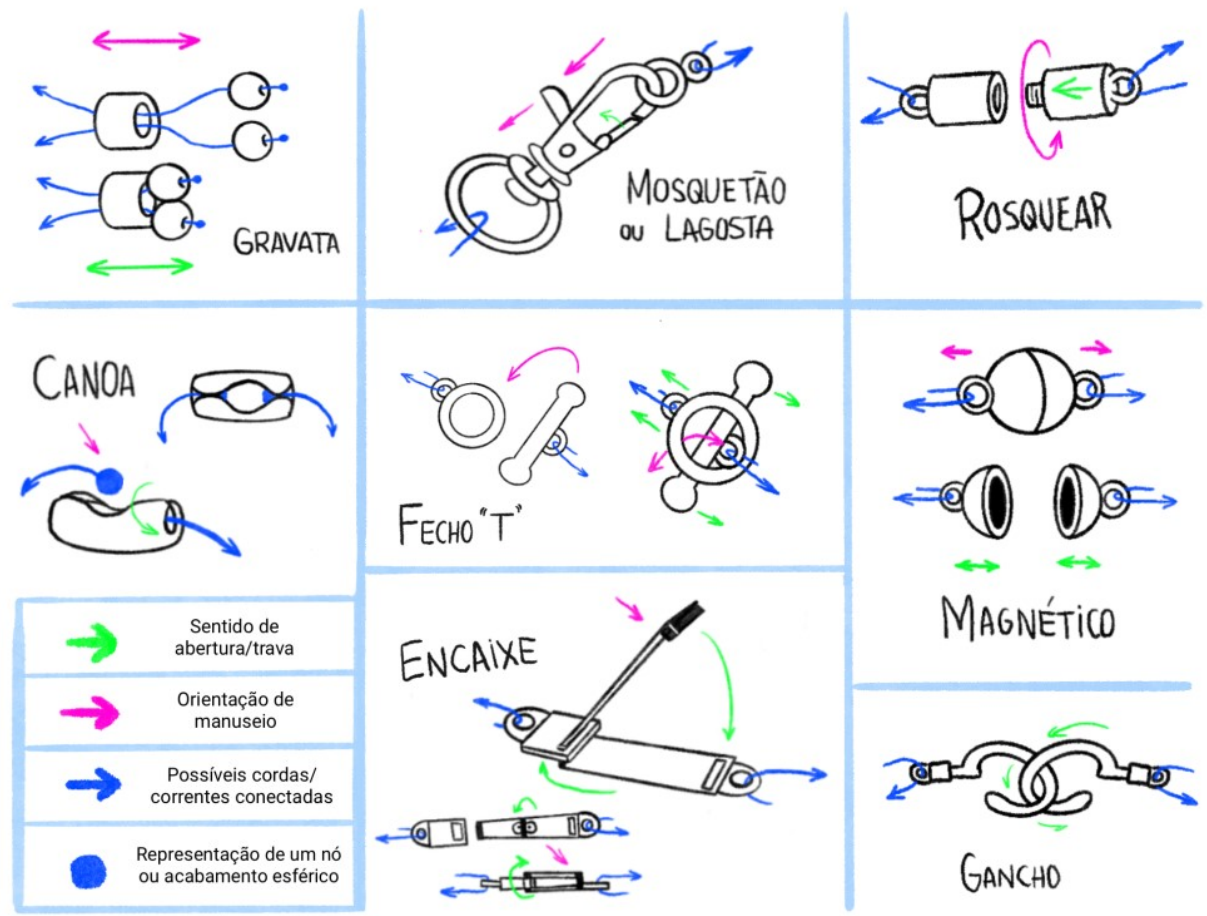

Fonte: Autoria própria (2019)

\section{CONSIDERAÇÕES FINAIS}

A presente pesquisa limitou-se à compreensão visual e catalogação de fechos de joalheria tradicional, geralmente forjados em metais ou materiais rígidos semelhantes. No percurso, foi perceptível o monopólio do conhecimento sobre fechos, presente grande parte em formato de literatura estrangeira e paga. Não sendo possível acessar tais leituras, foi realizada uma catalogação como uma maneira de fornecer subsídios ao projeto da coleção de joias.

A partir dessa compilação, projetos de naturezas diversas (não profissionais, iniciantes, exploratórios) poderão ser facilitados, pois a compreensão de mecanismos, assim como suas potencialidades e limitações, deve ser bem estabelecida para determinar a escolha e aplicação no processo projetual de joias. Por exemplo, um fecho magnético funciona muito melhor para uma pulseira, cujo manuseio é dificultado por ocorrer com uma só mão, enquanto pode não aguentar um colar pesado. É imprescindível o conhecimento geral de fechos para a produção de novos. Como observado, essa criação não precisa ser completamente inovadora mecanicamente, mas pode ser uma releitura em formas que nos leve a desassociar completamente do original, distinguindo e valorizando um produto. 
Diante da pandemia COVID-19 não foram possíveis testes físicos de impressão para avaliar a adaptação e funcionamento dos modelos (aqui catalogados) de material tradicional para a manufatura aditiva. A compreensão da reação deste e outros materiais também rendem novos estudos, que podem ainda ser realizados no âmbito da plataforma $\mathrm{MuVuCa}$, que se desdobrará como projeto de extensão universitária na UTFPR.

\section{REFERÊNCIAS}

LORENZ, Bruno Augusto. Pesquisa através do Design e Prática Crítica: uma investigação sobre o desenvolvimento de artefatos críticos no processo de construção de problemas de pesquisa acadêmicos. 2018. 205 f. Dissertação (Mestrado) - Curso de Design, Universidade do Vale do Rio dos Sinos, Porto Alegre, 2018.

FINDELLI, A. A Quest for Credibility: Doctoral Education and Research in Design at the University of Montreal. Doctoral Education in Design, Ohio, 8-11 October, 1998.

JONAS, Wolfgang. Design Research and its Meaning to the Methodological Development of the Discipline. In: MICHEL, Ralf (ed.). Design Research Now. Berlin: Birkhäuser Basel, 2007. p. 187-206.

DESCONHECIDO. Panda Hall. 2019. Disponível em:

https://www.pandahall.com/wholesale-

clasps/309.html?keyword=\%2Bjewelry\%20\%2Bclasps\&matchtype=b\&device=t\&g clid=CjwKCAjwyo36BRAXEiwA24CwGUiscTtTKejL3G I AQWauuaRTAsRweA4SL0a CPwDfhU1wdN9iNASRoCgBEQAvD BwE . Acesso em: 20 set. 2019.

DESCONHECIDO. Fabrique biju. 2019. Disponível em: https://www.fabriquebiju.com.br/pecas-de-montagem/fechos . Acesso em: 20 set. 2019.

DESCONHECIDO. Fazendo Arte Bijuterias. 2019. Disponível em: https://www.fazendoartebijuterias.com.br/pecas-para-montagem/fechos . Acesso em: 21 set. 2019.

DESCONHECIDO. Jewelry Supply. 2019. Disponível em: https://www.jewelrysupply.com/jewelry-clasps . Acesso em: 9 out. 2019.

DESCONHECIDO. Fire Mountain Gems. 2019. Disponível em: https://www.firemountaingems.com/resources/jewelry-making-articles/8b6h . Acesso em: 20 set. 2019. 
CHEW, Jane. Types of clasps for your jewelry. Disponível em: https://www.bloglovin.com/blogs/learn-to-make-handcrafted-jewelry4108449/types-clasps-for-your-jewelry-3740420963 . Acesso em: 20 set. 2019.

DESCONHECIDO. 14 Tipos de Fechos Folheados Ouro para Montagem de Semi Joias. 2018. Disponível em: http://blog.pedramistica.com.br/14-tipos-de-fechosfolheados-ouro-para-montagem-de-semi-joias/. Acesso em: 20 set. 2019.

LATTA, Amy. Perfectly Pair Your Projects With 8 Types of Jewelry Clasps. 2019. Disponível em: https://www.craftsy.com/post/types-of-jewelry-clasps/. Acesso em: 04 out. 2019.

RIVERA, Jose C. Locking Box Clasp For Jewelry. Procurador: Victor N. Sakran. US4881305A. Depósito: 29 abr. 1988. Concessão: 29 nov. 1989. Disponível em: https://patentimages.storage.googleapis.com/ac/34/b5/eb0c0cd27d61d1/US488 1305.pdf. Acesso em: 22 set. 2019.

COGDILL, Jolita. JEWELRY CLOSURE. Procurador: Robert J. Sandy. USA n. US6481065B2. Depósito: 15 fev. 2001. Concessão: 15 ago. 2002.Disponível em: https://patentimages.storage.googleapis.com/b8/3d/3c/4fe8784047eb8f/US648 1065.pdf. Acesso em: 22 set. 2019. 